\title{
Non-local spatial frequency response of photopolymer materials containing chain transfer agents: I. Theoretical modelling
}

\author{
Jinxin Guo, Michael R Gleeson, Shui Liu and John T Sheridan \\ School of Electrical, Electronic and Mechanical Engineering, Communications and \\ Optoelectronic Research Centre, The SFI-Strategic Research Cluster in Solar Energy \\ Conversion, College of Engineering, Mathematical and Physical Sciences, University College \\ Dublin, Belfield, Dublin 4, Ireland \\ E-mail: john.sheridan@ucd.ie and jinxin.guo@ucd.ie
}

Received 7 March 2011, accepted for publication 2 August 2011

Published 25 August 2011

Online at stacks.iop.org/JOpt/13/095601

\begin{abstract}
The non-local photopolymerization driven diffusion (NPDD) model predicts that a reduction in the non-local response length within a photopolymer material will improve its high spatial frequency response. The introduction of a chain transfer agent reduces the average molecular weight of polymer chains formed during free radical polymerization. Therefore a chain transfer agent (CTA) provides a practical method to reduce the non-local response length. An extended NPDD model is presented, which includes the chain transfer reaction and most major photochemical processes. The addition of a chain transfer agent into an acrylamide/polyvinyl alcohol photopolymer material is simulated and the predictions of the model are examined. The predictions of the model are experimentally examined in part II of this paper.
\end{abstract}

Keywords: holographic data storage, photopolymer material, chain transfer agents, spatial frequency response

(Some figures in this article are in colour only in the electronic version)

\section{Introduction}

Photopolymer materials are being actively studied for practical applications such as holographic data storage, hybrid optoelectronics, photo-embossing, including the manufacture of refractive and diffractive optical elements, and the selftrapping of light [1-4]. Generally, in photopolymers, the photosensitizer absorbs light of an appropriate wavelength, becoming excited and causing the production of primary radicals, $R^{*}$. In free radical polymerization systems, the generation of $R$. leads to monomers being polymerized. During holographic exposure, a grating is formed by the interference fringe pattern. The resulting polymer chains formed grow away from their point of initiation, which results in a loss of recording fidelity and a reduction in the refractive index modulation amplitude [5]. The nonlocal photopolymerization driven diffusion (NPDD) model was introduced to describe the observed decrease in the material's response for higher exposing spatial frequencies [6, 7]. The photopolymer's response at high spatial frequencies is very important as it determines the material's recording resolution and thus data storage capabilities. The NPDD model predicts that a reduction in the extent of the non-local effects within a material will result in an improvement in the high spatial frequency response, and this prediction has been previously studied and experimentally confirmed [7, 8].

Early models used to describe photopolymer performance [9-11] did not include the non-local effects, and did not predict the high spatial frequency roll-off. The NPDD model $[7,12,13]$ provides a more accurate physical description, which explains the high spatial frequency cutoff and the experimentally observed temporal evolution of holographic grating formation. In a free radical polymerization system, the physical and mechanical characteristics of the polymer chains formed depend on their molecular weight. The application of chain transfer agents (CTAs) as a means 
to control polymer chain length grown has therefore received much attention [14-17]. In this series of papers, we study in detail the effects of the addition of chain transfer agents into an acrylamide/polyvinyl alcohol (AA/PVA) based photopolymer material in order to improve its spatial frequency response. Our objective is to understand and fully quantify the performance and kinetics of the CTAs under examination.

In this first paper, part I, the NPDD model is extended to include the kinetic behaviour of CTAs during holographic grating formation in photopolymer materials. This paper is organized as follows. In section 2, we briefly review the photochemical processes occurring during holographic grating formation in an AA/PVA based photopolymer material and describe the chain transfer process in such a free radical based system. In section 3, the kinetic model governing the photochemistry is presented. Then the equations describing diffraction by holographic gratings recorded in these materials are reviewed. In section 4 , the kinetic behaviour of the reactions and the resulting material spatial frequency response are simulated using the developed model. Predictions for various parameter values are presented and compared. A brief conclusion is given in section 5 .

In part II [18], the results of a series of experiments are analysed using the model developed here in part I. Various concentrations of two different CTAs are included in the standard AA/PVA material and their effects on the average molecular length of the polyacrylamide (PA) chains formed during exposure are examined. Then the spatial frequency responses of these different materials are examined with and without the various concentrations of the chain transfer agents present, in order to determine CTA performance. The model is then used to estimate the physical parameters associated with both types of CTA used by fitting the modified NPDD model to the experimental results.

\section{Photochemical reactions}

In the case of free radical photopolymerization systems, the kinetic model describing what takes place involves five main processes: (1) initiation, (2) propagation, (3) termination, (4) inhibition, and (5) chain transfer, each of which may involve several physicochemical reactions. We highlight the major chemical reactions in each process below [19-23].

\section{(1) Initiation}

During illumination, the reaction between the photosensitizer and the electron donor (co-initiator) leads to the production of initiator radicals, $R^{*}$, which can react with the monomers to produce chain initiators, $M \cdot[21]$ :

$$
\begin{gathered}
I \stackrel{h v}{\longrightarrow} R^{*}, \\
R^{\cdot}+M \stackrel{k_{\mathrm{i}}}{\longrightarrow} M^{*},
\end{gathered}
$$

where $I$ is the initiator, $h v$ indicates the energy absorbed from a photon, $k_{\mathrm{i}}$ is the chain initiation kinetic constant and $M$ represents a monomer molecule. The absorption process, which occurs during the initiation step, will be discussed in more detail in section 3.1.

\section{(2) Propagation}

The chain initiator, $M_{i}$, will attach itself to another monomer molecule, $M$, by addition to the $\mathrm{C}=\mathrm{C}$ bond, yielding a growing polymer radical with an active tip. Through propagation the polymer chain grows [19]:

$$
M_{n}^{\cdot}+M \stackrel{k_{\mathrm{p}}}{\longrightarrow} M_{n+1}^{\cdot},
$$

where $k_{\mathrm{p}}$ is the rate constant of propagation and $M_{n}$ and $M_{n+1}^{\cdot}$ are the growing macro-radical chains of $n$ and $(n+1)$ repeat monomeric units $(n \geqslant 1)$.

\section{(3) Termination}

Termination can occur in three ways. Two of these, disproportionation and combination, involve two growing macro-radicals interacting, i.e., the bimolecular termination mechanism:

$$
\begin{gathered}
M_{n}^{\cdot}+M_{m}^{\cdot} \stackrel{k_{\mathrm{tc}}}{\longrightarrow} M_{n+m}, \\
M_{n}^{\cdot}+M_{m}^{\cdot} \stackrel{k_{\mathrm{td}}}{\longrightarrow} M_{n}+M_{m},
\end{gathered}
$$

where $k_{\mathrm{tc}}$ and $k_{\mathrm{td}}$ are the rate constants of combination and disproportionation termination respectively. $M_{n}, M_{m}$ and $M_{n+m}$ represent terminated chains which have no radical tip, i.e., a dead polymer. In this analysis, $k_{\mathrm{tc}}$ and $k_{\mathrm{td}}$ will be treated as a single lumped rate constant, $k_{\mathrm{t}}=k_{\mathrm{tc}}+k_{\mathrm{td}}\left(\mathrm{cm}^{3} \mathrm{~mol}^{-1} \mathrm{~s}^{-1}\right)$, as the mode of termination does not affect the polymerization kinetics [21].

A third possible termination mechanism involves primary radical termination $[21,24]$ :

$$
M_{n}^{\cdot}+R \stackrel{k_{\mathrm{tp}}}{\longrightarrow} M_{n} R
$$

where $k_{\mathrm{tp}}$ is the rate constant of primary radical termination. In this step, a growing macro-radical chain reacts with a primary radical (initiator radical) leading once again to the production of inactive or dead polymer chains [21].

\section{(4) Inhibition}

Inhibitors are chemicals which react with the initiating and propagating radical species by rapidly removing or scavenging these radicals. Polymerization is completely halted until they are all consumed [20]. Several possible inhibitor reaction mechanisms are listed below:

$$
\begin{gathered}
\text { Dye }^{*}+Z \stackrel{k_{z, \mathrm{D}}}{\longrightarrow} \text { Leuco Dye }+Z^{*}, \\
R^{*}+Z \stackrel{k_{z, R^{*}}}{\longrightarrow}\left(R+Z^{*}, \text { and/or } R Z^{*}\right), \\
M_{n}^{\cdot}+Z \stackrel{k_{z, M^{*}}}{\longrightarrow}\left(M_{n}+Z^{*}, \text { and/or } M_{n} Z^{*}\right),
\end{gathered}
$$

where $Z$ is the inhibitor species, e.g., oxygen, dye* is the excited photosensitizer, $Z^{*}$ is the concentration of singlet oxygen $[20,23-25]$, and $k_{z, \mathrm{D}}, k_{z, M}$, and $k_{z, R}$ are the rate constants of inhibition of the photosensitizer, the macroradicals and the primary radicals respectively.

Inhibition leads to a dead band at the start exposure, i.e., no initial grating formation during exposure. The effects of inhibitors are especially significant when lower exposure intensities are used, for example when large areas must be exposed or short pulses must be used [23]. In order to 
further simplify the reaction modelling, we assume that $Z^{*}$, $R Z^{\cdot}$ and $M_{n} Z^{*}$ do not re-initiate polymerization and that they terminate without regeneration $[21,23]$. In addition, $Z \cdot R-$ $Z \cdot$ and $M_{n}-Z^{\cdot}$ are assumed to play no further part in grating formation, i.e., no further chain transfer mechanisms involve them. Furthermore, in the sealed material layer, it is assumed that the inhibitors are rapidly consumed at the start of the exposure [26].

\section{(5) Chain transfer mechanism}

In many polymerization systems, the average polymer weight is observed to be lower than that predicted by the chain transfer reaction [8, 14-17, 20,27]. Generally, the chain transfer process causes the premature termination of a growing macro-radical chain and arises because of the presence of a CTA [20]. Due to this reaction, a new radical is produced which is referred to as a re-initiator. This re-initiator reacts with a monomer molecule to initiate a new growing macroradical chain. The chain transfer reactions can be written as

$$
\begin{gathered}
M_{n}^{\cdot}+(R I-X) \stackrel{k_{\mathrm{tr}, \mathrm{S}}}{\longrightarrow}\left(M_{n}-X\right)+R I^{\cdot}, \\
R I^{\cdot}+M \stackrel{k_{\mathrm{ri}}}{\longrightarrow}\left(R I-M^{\cdot}\right),
\end{gathered}
$$

where $R I-X$ is the chain transfer agent, $-X$ is the atom or species transferred and $R I^{*}$ is the re-initiator which has a radical tip. $k_{\mathrm{tr}, \mathrm{S}}$ and $k_{\mathrm{ri}}$ are the transfer rate constant to chain transfer agent and the re-initiation rate constant respectively. Due to the premature termination reaction with the chain transfer agent, $R I-X$, the propagating polymer chains will stop growing earlier than they would have if the CTA was not present. We assume that the free radical $R I-$ $M \cdot$ produced can be treated as acting chemically identically to a chain initiator $M^{*}$. Therefore the re-initiator, $R I^{*}$, simply initiates a new growing chain with a radical tip $M^{*}$. Thus, while the polymer chains are shortened, the amount of monomer polymerized and the rate of polymerization can remain high.

\section{Photochemical kinetic model}

\subsection{Absorption}

During holographic grating formation in the AA/PVA based photopolymer, photosensitization and initiation depend on the type and concentrations of both the dye and electron donor. During appropriate illumination of such a layer, sensitized with a xanthene or thiazine type dye [19, 26], the photosensitizer absorbs a photon and is promoted into an excited state. As described in equation (1), an initiating species $R^{\cdot}$ is produced by the reaction between an excited dye molecule and an electron donor molecule (triethanolamine (TEA), $\mathrm{C}_{6} \mathrm{H}_{15} \mathrm{NO}_{3}$ ) [19]. The major reactions taking place during this step are as follows [23]:

$$
\begin{gathered}
\text { Dye }+h v \stackrel{k_{\mathrm{a}}}{\longrightarrow} \text { Dye }^{*}, \\
\text { Dye }^{*}+Z \stackrel{k_{z, \mathrm{D}}}{\longrightarrow} \text { Leuco Dye, } \\
\text { Dye }^{*} \stackrel{k_{\mathrm{r}}}{\longrightarrow} \text { Dye, }
\end{gathered}
$$

$$
\begin{gathered}
\text { Dye }^{*}+E D \stackrel{k_{\mathrm{d}}}{\longrightarrow} R^{*}+\mathrm{H}^{+}+\mathrm{Dye}^{-} \longrightarrow R^{*}+\text { HDye } \\
E D+\mathrm{HDye} \stackrel{k_{\mathrm{b}}}{\longrightarrow} \mathrm{H}_{2} \text { Dye }+E D_{\text {int }},
\end{gathered}
$$

where Dye represents the photosensitizer (dye) species, ED is the co-initiator (electron donor) and $Z$ is the inhibitor. HDye' represents a radicalized dye, which has abstracted a hydrogen ion from the co-initiator, and $\mathrm{H}_{2}$ Dye is the di-hydro transparent form of the dye, i.e., a bleached transparent form of the dye molecule. $E D_{\text {int }}$ is an intermediate form of the co-initiator [19]. $\quad k_{\mathrm{a}}\left(\mathrm{s}^{-1}\right)$ is the rate of production of excited state photosensitizer; $k_{z, \mathrm{D}}$ $\left(\mathrm{cm}^{3} \mathrm{~mol}^{-1} \mathrm{~s}^{-1}\right)$ is the inhibition rate constant associated with the reaction of the inhibitor with the excited dye molecules, i.e., the formation of Leuco Dye; $k_{\mathrm{r}}\left(\mathrm{s}^{-1}\right)$ is the rate of recovery or regeneration of the photosensitizer back to the ground (absorbing) state; $k_{\mathrm{d}}\left(\mathrm{cm}^{3} \mathrm{~mol}^{-1} \mathrm{~s}^{-1}\right)$ is the rate of dissociation of the initiator; and $k_{\mathrm{b}}$ is the rate constant of bleaching. In equation $(6 d)$, the dye radical formed abstracts a hydrogen molecule from the TEA free radical.

Applying the Beer-Lambert law, the rate of production of the excited state photosensitizer in equation $(6 a)$ can be expressed as $k_{\mathrm{a}}=\phi \varepsilon d I_{0}^{\prime}\left(\mathrm{s}^{-1}\right)[23,26]$, where $\phi$ (mol/Einstein) is the quantum efficiency of the reaction, $\varepsilon$ $\left(\mathrm{cm}^{2} \mathrm{~mol}^{-1}\right)$ is the molar absorptivity coefficient of the dye, $d$ is the material layer thickness and $I_{0}^{\prime}$ (Einstein $\mathrm{cm}^{-2} \mathrm{~s}^{-1}$ ) is the incident intensity corrected for Fresnel and scattering losses using the factor $T_{\mathrm{sf}}$. The exposure intensity $I_{0}$ is typically measured in units of $\mathrm{mW} \mathrm{cm}^{-2}$, and must be converted into units of Einstein $\mathrm{cm}^{-2} \mathrm{~s}^{-1}$. This conversion can be performed using $I_{0}^{\prime}=\frac{T_{\mathrm{sf}} B I_{0}}{d}\left(\frac{\lambda}{N_{\mathrm{a}} h c}\right)$, where $\lambda$ (nm) is the wavelength of incident light, $N_{\mathrm{a}}\left(\mathrm{mol}^{-1}\right)$ is Avogadro's constant, $c\left(\mathrm{~m} \mathrm{~s}^{-1}\right)$ is the speed of light, and $h(\mathrm{~J} \mathrm{~s})$ is Plank's constant. $\quad B=1-\mathrm{e}^{-\varepsilon A_{0} d}$ is the absorptive fraction which determines a material layer's initial absorptive capacity and is a function of the dye's initial concentration, $\left[A_{0}\right]\left(\mathrm{mol} \mathrm{cm}^{-3}\right)$ [23]. The evolution of the concentration of the excited state dye, Dye*, can be determined for a given exposure intensity and initial photosensitizer concentration assuming $\phi, \varepsilon, d$ and $T_{\text {sf }}$ are known.

\subsection{NPDD model}

We assume illumination by a spatial distribution of irradiance $I(x, t)=I_{0}^{\prime}[1+V \cos (K x)]$, where $V$ is the fringe visibility and $K=2 \pi / \Lambda$, where $\Lambda$ is the grating period $[9,28]$. The chemical equations, presented in equation (6), describe in detail primary radical production, which was previously summarized in section 2 . We now derive a set of coupled differential equations representing the spatial and temporal evolutions of the material concentrations associated with equation (6). Combining these with those previously presented in $[23,26]$, a more complete set of governing rate equations is now presented:

$\frac{\mathrm{d}[\operatorname{Dye}(x, t)]}{\mathrm{d} t}=-k_{\mathrm{a}}[\operatorname{Dye}(x, t)]+k_{\mathrm{r}}\left[\operatorname{Dye}^{*}(x, t)\right]$, 


$$
\begin{aligned}
& \frac{\mathrm{d}\left[\operatorname{Dye}^{*}(x, t)\right]}{\mathrm{d} t}=k_{\mathrm{a}}[\operatorname{Dye}(x, t)]-k_{\mathrm{r}}\left[\operatorname{Dye}^{*}(x, t)\right] \\
& -k_{\mathrm{d}}\left[\operatorname{Dye}^{*}(x, t)\right][E D(x, t)] \\
& -k_{z, \mathrm{D}}\left[\mathrm{Dye}^{*}(x, t)\right][Z(x, t)] \text {, } \\
& \frac{\mathrm{d}[E D(x, t)]}{\mathrm{d} t}=-k_{\mathrm{d}}\left[\operatorname{Dye}^{*}(x, t)\right][\mathrm{ED}(x, t)] \\
& -k_{\mathrm{b}}[\operatorname{HDye}(x, t)][E D(x, t)], \\
& \frac{\mathrm{d}\left[\operatorname{HDye}^{\cdot}(x, t)\right]}{\mathrm{d} t}=k_{\mathrm{d}}\left[\operatorname{Dye}^{*}(x, t)\right][E D(x, t)] \\
& -k_{\mathrm{b}}\left[\operatorname{HDye}^{\cdot}(x, t)\right][E D(x, t)], \\
& \frac{\mathrm{d}[Z(x, t)]}{\mathrm{d} t}=\frac{\mathrm{d}}{\mathrm{d} x}\left\{D_{Z}(x, t) \frac{\mathrm{d}[Z(x, t)]}{\mathrm{d} x}\right\} \\
& -k_{z, \mathrm{D}}\left[\mathrm{Dye}^{*}(x, t)\right][Z(x, t)]-k_{z, R} \cdot[Z(x, t)]\left[R^{\cdot}(x, t)\right] \\
& -k_{z, M} \cdot[Z(x, t)]\left[M^{\cdot}(x, t)\right]+\tau_{z}\left\{Z_{0}-[Z(x, t)]\right\} .
\end{aligned}
$$

As indicated in equation (11), the non-uniform recording irradiance gives rise to concentration gradients, which result in the diffusion of inhibitor from the dark regions into the bright illuminated regions. Equation (11) is derived based on the assumption that the oxygen is relatively free to diffuse, and does so rapidly, resulting in a one-dimensional standard diffusion equation for the concentration of inhibitor [23, 29]. As in the previous analysis [21-23], in equation (11) it is assumed that the effect of inhibition during exposure is caused by the initially dissolved oxygen present within the photopolymer layer and replenishing oxygen from the surrounding environment. In such an unsealed material layer case we introduced the parameter $\tau_{z}$ to represent the rate of replenishing of oxygen into the material layer from the surrounding environment. Also, we assume that $k_{z}=k_{z, R^{*}}=$ $k_{z, M}$ and $k_{z, \mathrm{D}} \ll k_{z}$, as the reactivity of oxygen with the excited state form of the photosensitizer will be much lower than with $R^{\cdot}$ or $M^{\cdot}[20]$. [Z(x,t)] is the instantaneous inhibitor concentration, $D_{Z}(x, t)$ is the diffusion rate of the inhibitor in the dry material layer, and $Z_{0}\left(\mathrm{~mol} \mathrm{~cm}^{-3}\right)$ is the initial dissolved inhibitor concentration. In addition, the diffusion of dye molecules (due to the concentration gradient) from the dark regions into the bright regions will eventually take place over an appreciably long time [30]. However, clearly the dye concentration following such diffusion cannot be larger than its initial value. We assume that the effects of dye diffusion are negligible in this paper.

The equation governing the concentration of primary radicals is given by

$$
\begin{aligned}
& \frac{\mathrm{d}\left[R^{\cdot}(x, t)\right]}{\mathrm{d} t}=k_{\mathrm{d}}\left[\operatorname{Dye}^{*}(x, t)\right][E D(x, t)] \\
& \quad-k_{\mathrm{i}}\left[R^{\cdot}(x, t)\right][u(x, t)]-k_{\mathrm{tp}}\left[R^{\cdot}(x, t)\right]\left[M^{\cdot}(x, t)\right] \\
& \quad-k_{z}\left[R^{\cdot}(x, t)\right][Z(x, t)],
\end{aligned}
$$

where $u(x, t)$ is the free-monomer concentration. This equation states that the contributions to the rate of change of the primary radical concentration include the generation of primary radicals by photon absorption, and the amounts removed by (a) the initiation of macro-radicals, (b) primary termination with growing polymer chains, and (c) inhibition by oxygen.
The equation governing the macro-radical concentration is then

$$
\begin{aligned}
& \frac{\mathrm{d}\left[M^{\cdot}(x, t)\right]}{\mathrm{d} t}=k_{\mathrm{i}}\left[R^{\cdot}(x, t)\right][u(x, t)]-k_{\mathrm{t}}\left[M^{\cdot}(x, t)\right]^{2} \\
& \quad-k_{\mathrm{tp}}\left[R^{\cdot}(x, t)\right]\left[M^{\cdot}(x, t)\right]-k_{z}[Z(x, t)]\left[M^{\cdot}(x, t)\right] .
\end{aligned}
$$

The generation term in this equation previously appears as the removal term, arising due to macro-radical initiation, in equation (12). The removal terms include the effects of both types of termination mechanism, i.e., primary and bimolecular (the squared term), and the effects of inhibition [21-23].

The co-sinusoidal irradiance leads to monomer concentration gradients, that result in monomer diffusion from the dark regions into the monomer depleted exposed regions. The polymer concentration distribution produced by the exposure provides the permanent modulation of refractive index in the material, i.e., the holographic grating. We represent the monomer concentration using the following 1D equation [23]:

$$
\begin{aligned}
& \frac{\mathrm{d}[u(x, t)]}{\mathrm{d} t}=\frac{\mathrm{d}}{\mathrm{d} x}\left\{D_{\mathrm{m}}(x, t) \frac{\mathrm{d}[u(x, t)]}{\mathrm{d} x}\right\}-k_{\mathrm{i}}\left[R^{\cdot}(x, t)\right] \\
& \quad \times[u(x, t)]-\int_{-\infty}^{\infty} k_{\mathrm{p}}\left[M^{\cdot}\left(x^{\prime}, t\right)\right]\left[u\left(x^{\prime}, t\right)\right] G\left(x, x^{\prime}\right) \mathrm{d} x^{\prime}
\end{aligned}
$$

where $D_{m}(x, t)$ represents the monomer diffusion rate, and $G\left(x, x^{\prime}\right)$ is the non-local material spatial response function given by [7]

$$
G\left(x, x^{\prime}\right)=\frac{1}{\sqrt{2 \pi \sigma}} \exp \left[\frac{-\left(x-x^{\prime}\right)^{2}}{2 \sigma}\right],
$$

in which $\sigma$ is the non-local response parameter. The nonlocal spatial response function represents the effect of initiation at a location $x^{\prime}$ on the amount of monomer polymerized at location $x[6-8,21-23,26,31,32] . \sqrt{\sigma}$ represents the nonlocal response length, which is related to the average polymer chain length [31].

The equation governing the polymer concentration is

$$
\begin{aligned}
& \frac{\mathrm{d}[N(x, t)]}{\mathrm{d} t}=-\frac{\mathrm{d}}{\mathrm{d} x}\left\{D_{N}(x, t) \frac{\mathrm{d}[N(x, t)]}{\mathrm{d} x}\right\} \\
& +\int_{-\infty}^{\infty} k_{\mathrm{p}}\left[M\left(x^{\prime}, t\right)\right]\left[u\left(x^{\prime}, t\right)\right] G\left(x, x^{\prime}\right) \mathrm{d} x^{\prime},
\end{aligned}
$$

where $D_{N}(x, t)$ represents the polymer diffusion rate. If the polymer chains are not cross-linked sufficiently, they will tend to diffuse out of the exposed regions where they are formed [8]. This process will result in a decay of the grating strength with time. However, in this paper we assume that there is sufficient cross-linking and therefore $D_{N}(x, t)=0$, i.e., very stable gratings are formed.

In the radical chain polymerization system $[8,20]$, the polymerization rate can be expressed as

$$
R_{\mathrm{P}}=k_{\mathrm{p}}[M \cdot(x, t)][u(x, t)] .
$$


The polymerization rate, $R_{\mathrm{p}}$, is also related to the numberaverage degree of polymerization, $D P_{n} . D P_{n}$ is defined as the average number of structural units per polymer chain. It indicates the average length and therefore molecular weight of the polymer chains formed. According to the Mayo equation $[14,20]$

$$
\frac{1}{D P_{n}}=\frac{k_{\mathrm{t}} R_{\mathrm{p}}}{k_{\mathrm{p}}^{2}[u]^{2}}+C_{\mathrm{u}}+C_{\mathrm{CTA}} \frac{[C T A]}{[u]}+C_{I} \frac{[I]}{[u]} .
$$

This quantifies the effect of the various chain transfer reactions on the number-average degree of polymerization. $[u]$, [CT A], and $[I]$, represent the concentrations of monomer, chain transfer agent and initiator, respectively. The chain transfer constants, $C_{\mathrm{u}}, C_{\mathrm{CTA}}$ and $C_{\mathrm{I}}$, for each particular substance are defined as the ratios of the rate constants for chain transfer of a propagating radical with that substance to the propagation rate constant, $k_{\mathrm{p}}$. They are given by

$$
\begin{gathered}
C_{\mathrm{u}}=\frac{k_{\mathrm{tr}, \mathrm{u}}}{k_{\mathrm{p}}}, \quad C_{\mathrm{CTA}}=\frac{k_{\mathrm{tr}, \mathrm{CTA}}}{k_{\mathrm{p}}}, \quad \text { and } \\
C_{I}=\frac{k_{\mathrm{tr}, \mathrm{I}}}{k_{\mathrm{p}}}
\end{gathered}
$$

where $k_{\mathrm{tr}, \mathrm{u}}, k_{\mathrm{tr}, \mathrm{CTA}}, k_{\mathrm{tr}, \mathrm{I}}$ represent rate constants for chain transfer to monomer, chain transfer agent and initiator respectively. For the case examined here the chain transfer constants to monomer and initiator can be omitted as they are typically very low for acrylamide [33, 34], and therefore equation (18) can be simplified to

$$
\frac{1}{D P_{n}}=\frac{k_{\mathrm{t}} R_{\mathrm{p}}}{k_{\mathrm{p}}^{2}[u]^{2}}+C_{\mathrm{CTA}} \frac{[C T A]}{[u]},
$$

which will be discussed in detail in section 3.3.

\subsection{Model development}

In order to begin to examine the effects of the presence of CTA on the material non-local response length, $\sqrt{\sigma}$, we introduce a rate equation governing the CTA concentration:

$$
\begin{aligned}
& \frac{\mathrm{d}[C T A(x, t)]}{\mathrm{d} t}=\frac{\mathrm{d}}{\mathrm{d} x}\left\{D_{\mathrm{CTA}}(x, t) \frac{\mathrm{d}[C T A(x, t)]}{\mathrm{d} x}\right\} \\
& -k_{\mathrm{tr}, \mathrm{S}}[C T A(x, t)]\left[M^{*}(x, t)\right] .
\end{aligned}
$$

It should be noted that in the following analysis, which is based on the assumptions stated earlier in sections 2 and 3, we only consider chain transfer to the chain transfer agent. To further simplify the analysis in this paper, we assume that $k_{\mathrm{tr}}=k_{\mathrm{tr}, \mathrm{CTA}}$ and that the CTA diffusion rate, $D_{\mathrm{CTA}}$, is similar to the diffusion rate of monomer, $D_{\mathrm{m}}$, as their molecular weights are similar in the cases examined, i.e., $D_{\mathrm{CTA}} \approx D_{\mathrm{m}}$.

The equation governing the re-initiator concentration is

$$
\begin{aligned}
& \frac{\mathrm{d}\left[R I^{\cdot}(x, t)\right]}{\mathrm{d} t}=k_{\mathrm{tr}}[C T A(x, t)][M \cdot(x, t)] \\
& -k_{\mathrm{ri}}\left[R I^{\cdot}(x, t)\right][u(x, t)],
\end{aligned}
$$

where $R I^{\cdot}$ denotes the re-initiator concentration. The generation term here is the removal term arising in equation (5) due to the chain transfer process. Since it is assumed that the initiator radical, $R^{\cdot}$, dominates the primary termination and inhibition processes, we only consider how the re-initiator, $R I^{\circ}$, reacts with the monomer.

Furthermore the chain transfer and re-initiation reactions affect the variation of macro-radical, $\left[M^{*}\right]$, and monomer, $[u]$, concentrations. Therefore equations (13) and (14) must be generalized:

$$
\begin{aligned}
& \frac{\mathrm{d}\left[M^{\cdot}(x, t)\right]}{\mathrm{d} t}=k_{\mathrm{i}}\left[R^{\cdot}(x, t)\right][u(x, t)]+k_{\mathrm{ri}}\left[R I^{\cdot}(x, t)\right][u(x, t)] \\
& \quad-k_{\mathrm{t}}\left[M^{\cdot}(x, t)\right]^{2}-k_{\mathrm{tp}}\left[R^{\cdot}(x, t)\right]\left[M^{\cdot}(x, t)\right] \\
& -k_{z}[Z(x, t)]\left[M^{\cdot}(x, t)\right]-k_{\mathrm{tr}}[C T A(x, t)]\left[M^{\cdot}(x, t)\right],
\end{aligned}
$$

$$
\begin{aligned}
& \frac{\mathrm{d}[u(x, t)]}{\mathrm{d} t}=\frac{\mathrm{d}}{\mathrm{d} x}\left\{D_{\mathrm{m}}(x, t) \frac{\mathrm{d}[u(x, t)]}{\mathrm{d} x}\right\}-k_{\mathrm{i}}\left[R^{\cdot}(x, t)\right] \\
& \quad \times[u(x, t)]-\int_{-\infty}^{\infty} k_{\mathrm{p}}\left[M^{\cdot}\left(x^{\prime}, t\right)\right]\left[u\left(x^{\prime}, t\right)\right] G\left(x, x^{\prime}\right) \mathrm{d} x^{\prime} \\
& \quad-k_{\mathrm{ri}}\left[R I^{\cdot}(x, t)\right][u(x, t)] .
\end{aligned}
$$

All the concentrations appearing in equations (7)-(12) and (16), (21)-(24), will be periodic even functions of $x$ and can therefore be written as Fourier series, i.e., $[X(x, t)]=$ $\sum_{j=0}^{\infty} X_{j}(t) \cos (j K x)$, where $X$ represents the particular species, i.e., Dye, Dye* $E D$, HDye', $R \cdot M \cdot C T A, R I$, $u$, $N$ or $Z$. A set of first-order coupled differential equations can then be obtained by gathering the coefficients of the various co-sinusoidal spatial components and writing the equations in terms of these time varying spatial harmonic amplitudes, $X_{j}(t)$. Assuming that harmonics of order greater than $j=3$ are negligible, equations (25)-(29) can be obtained in the same manner as described in [21-23]. For brevity, we only present the coupled equations for the first two harmonics of [CT A], $\left[R I^{\cdot}\right],\left[M^{*}\right]$, and $[u]$, all of which are directly involved in reactions with the transfer agent.

3.3.1. Chain transfer agent concentration. Retaining the first four concentration harmonic amplitudes in the analysis, the following first-order coupled differential equations govern the chain transfer agent concentration amplitudes, $C T A_{j}$ :

$$
\begin{aligned}
& \frac{\mathrm{d} C T A_{0}(t)}{\mathrm{d} t}=-k_{\mathrm{tr}} C T A_{0}(t) M_{0}(t)-\frac{1}{2} k_{\mathrm{tr}} C T A_{1}(t) M_{1}(t) \\
& \quad-\frac{1}{2} k_{\mathrm{tr}} C T A_{2}(t) M_{2}(t)-\frac{1}{2} k_{\mathrm{tr}} C T A_{3}(t) M_{3}(t) \\
& \frac{\mathrm{d} C T A_{1}(t)}{\mathrm{d} t}=-D_{\mathrm{CTA}} K^{2} C T A_{1}(t)-k_{\mathrm{tr}} C T A_{1}(t) M_{0}(t) \\
& \quad-k_{\mathrm{tr}} C T A_{0}(t) M_{1}(t)-\frac{1}{2} k_{\mathrm{tr}} C T A_{2}(t) M_{1}(t) \\
& \quad-\frac{1}{2} k_{\mathrm{tr}} C T A_{1}(t) M_{2}(t)-\frac{1}{2} k_{\mathrm{tr}} C T A_{3}(t) M_{2}(t) \\
& -\frac{1}{2} k_{\mathrm{tr}} C T A_{2}(t) M_{3}(t) .
\end{aligned}
$$

3.3.2. Re-initiator concentration. The equations governing the re-initiator concentration amplitudes, $R I_{j}^{*}$, are

$$
\begin{aligned}
& \frac{\mathrm{d} R I_{0}(t)}{\mathrm{d} t}=k_{\mathrm{tr}} C T A_{0}(t) M_{0}(t)+\frac{1}{2} k_{\mathrm{tr}} C T A_{1}(t) M_{1}(t) \\
& \quad+\frac{1}{2} k_{\mathrm{tr}} C T A_{2}(t) M_{2}(t)+\frac{1}{2} k_{\mathrm{tr}} C T A_{3}(t) M_{3}(t) \\
& \quad-k_{\mathrm{ri}} R I_{0}(t) u_{0}(t)-\frac{1}{2} k_{\mathrm{ri}} R I_{1}(t) u_{1}(t) \\
& \quad-\frac{1}{2} k_{\mathrm{ri}} R I_{2}^{\cdot}(t) u_{2}(t)-\frac{1}{2} k_{\mathrm{ri}} R I_{3}(t) u_{3}(t),
\end{aligned}
$$




$$
\begin{aligned}
& \frac{\mathrm{d} R I_{1}(t)}{\mathrm{d} t}=k_{\mathrm{tr}} C T A_{1}(t) M_{0}(t)+k_{\mathrm{tr}} C T A_{0}(t) M_{1}(t) \\
& \quad+\frac{1}{2} k_{\mathrm{tr}} C T A_{2}(t) M_{1}(t)+\frac{1}{2} k_{\mathrm{tr}} C T A_{1}(t) M_{2}(t) \\
& \quad+\frac{1}{2} k_{\mathrm{tr}} C T A_{3}(t) M_{2}(t) \\
& \quad+\frac{1}{2} k_{\mathrm{tr}} C T A_{2}(t) M_{3}(t)-k_{\mathrm{ri}} R I_{1}(t) u_{0}(t)-k_{\mathrm{ri}} R I_{0}(t) u_{1}(t) \\
& \quad-\frac{1}{2} k_{\mathrm{ri}} R I_{2}^{\cdot}(t) u_{1}(t)-\frac{1}{2} k_{\mathrm{ri}} R I_{1}^{\cdot}(t) u_{2}(t) \\
& \quad-\frac{1}{2} k_{\mathrm{ri}} R I_{3}^{\cdot}(t) u_{2}(t)-\frac{1}{2} k_{\mathrm{ri}} R I_{2}(t) u_{3}(t) .
\end{aligned}
$$

3.3.3. Macro-radical concentration. From equation (23), the equations governing the $M_{j}$ are

$$
\begin{aligned}
& \frac{\mathrm{d} M_{0}(t)}{\mathrm{d} t}=-\frac{k_{\mathrm{tr}}}{2}\left[2 C T A_{0}(t) M_{0}(t)+C T A_{1}(t) M_{1}(t)\right. \\
& \left.+C T A_{2}(t) M_{2}(t)+C T A_{3}(t) M_{3}(t)\right] \\
& -\frac{k_{\mathrm{t}}}{2}\left[2 M_{0}(t)^{2}+M_{1}(t)^{2}+M_{2}(t)^{2}+M_{3}(t)^{2}\right] \\
& -\frac{k_{\mathrm{tp}}}{2}\left[2 M_{0}(t) R_{0}^{*}(t)+M_{1}(t) R_{1}^{*}(t)+M_{2}(t) R_{2}^{*}(t)\right. \\
& \left.+M_{3}(t) R_{3}^{\cdot}(t)\right]-\frac{k_{z}}{2}\left[2 M_{0}(t) Z_{0}(t)+M_{1}(t) Z_{1}(t)\right. \\
& \left.+M_{2}(t) Z_{2}(t)+M_{3}(t) Z_{3}(t)\right] \\
& +\frac{k_{\mathrm{i}}}{2}\left[2 R_{0}^{\cdot}(t) u_{0}(t)+R_{1}^{\cdot}(t) u_{1}(t)+R_{2}^{\cdot}(t) u_{2}(t)\right. \\
& \left.+R_{3}^{\cdot}(t) u_{3}(t)\right]+\frac{k_{\mathrm{ri}}}{2}\left[2 R I_{0}(t) u_{0}(t)+R I_{1}(t) u_{1}(t)\right. \\
& \left.+R I_{2}^{\cdot}(t) u_{2}(t)+R I_{3}^{\cdot}(t) u_{3}(t)\right], \\
& \frac{\mathrm{d} M_{1}(t)}{\mathrm{d} t}=-\frac{k_{\mathrm{tr}}}{2}\left[2 C T A_{1}(t) M_{0}(t)+2 C T A_{0}(t) M_{1}(t)\right. \\
& +C T A_{2}(t) M_{1}(t)+C T A_{1}(t) M_{2}(t)+C T A_{3}(t) M_{2}(t) \\
& \left.+C T A_{2}(t) M_{3}^{\cdot}(t)\right]-k_{\mathrm{t}}\left[2 M_{0}^{\cdot}(t) M_{1}(t)+M_{1}(t) M_{2}(t)\right. \\
& \left.+M_{2}(t) M_{3}(t)\right]-\frac{k_{\mathrm{tp}}}{2}\left[2 M_{1}(t) R_{0}^{*}(t)+2 M_{0}(t) R_{1}^{*}(t)\right. \\
& +M_{2}(t) R_{1}^{\cdot}(t)+M_{1}(t) R_{2}^{\cdot}(t)+M_{3}(t) R_{2}^{\cdot}(t) \\
& \left.+M_{2}(t) R_{3}(t)\right]-\frac{k_{z}}{2}\left[2 M_{1}(t) Z_{0}(t)+2 M_{0}(t) Z_{1}(t)\right. \\
& +M_{2}(t) Z_{1}(t)+M_{1}(t) Z_{2}(t)+M_{3}(t) Z_{2}(t) \\
& \left.+M_{2}(t) Z_{3}(t)\right]+\frac{k_{\mathrm{i}}}{2}\left[2 R_{1}(t) u_{0}(t)+2 R_{1}(t) u_{0}(t)\right. \\
& +R_{2}^{\cdot}(t) u_{1}(t)+R_{1}^{\cdot}(t) u_{2}(t)+R_{3}^{\cdot}(t) u_{2}(t) \\
& \left.+R_{2}^{\cdot}(t) u_{3}(t)\right]+\frac{k_{\mathrm{ri}}}{2}\left[2 R I_{1}(t) u_{0}(t)+2 R I_{1}(t) u_{0}(t)\right. \\
& +R I_{2}(t) u_{1}(t)+R I_{1}(t) u_{2}(t) \\
& \left.+R I_{3}^{\cdot}(t) u_{2}(t)+R I_{2}(t) u_{3}(t)\right] \text {. }
\end{aligned}
$$

3.3.4. Monomer concentration. The coupled equations for the monomer concentration harmonics, $u_{j}$, are

$$
\begin{aligned}
& \frac{\mathrm{d} u_{0}(t)}{\mathrm{d} t}=-\frac{k_{\mathrm{p}}}{2}\left[2 M_{0}(t) u_{0}(t)+M_{1}(t) u_{1}(t)+M_{2}(t) u_{2}(t)\right. \\
& \left.\quad+M_{3}(t) u_{3}(t)\right]-\frac{k_{\mathrm{i}}}{2}\left[2 R_{0}^{\cdot}(t) u_{0}(t)+R_{1}(t) u_{1}(t)\right. \\
& \left.\quad+R_{2}^{\cdot}(t) u_{2}(t)+R_{3}^{\cdot}(t) u_{3}(t)\right]-\frac{k_{\mathrm{ri}}}{2}\left[2 R I_{0}(t) u_{0}(t)\right. \\
& \left.\quad+R I_{1}^{\cdot}(t) u_{1}(t)+R I_{2}(t) u_{2}(t)+R I_{3}(t) u_{3}(t)\right],
\end{aligned}
$$

$$
\begin{aligned}
& \frac{\mathrm{d} u_{1}(t)}{\mathrm{d} t}=-\frac{S_{1} k_{\mathrm{p}}}{2}\left[2 M_{1}(t) u_{0}(t)+2 M_{0}(t) u_{1}(t)+M_{2}(t) u_{1}(t)\right. \\
& \left.\quad+M_{1}(t) u_{2}(t)+M_{3}(t) u_{2}(t)+M_{2}(t) u_{3}(t)\right] \\
& \quad-\frac{k_{\mathrm{i}}}{2}\left[2 R_{1}^{\cdot}(t) u_{0}(t)+2 R_{0}^{\cdot}(t) u_{1}(t)+R_{2}^{\cdot}(t) u_{1}(t)\right. \\
& \left.\quad+R_{1}^{\cdot}(t) u_{2}(t)+R_{3}^{\cdot}(t) u_{2}(t)+R_{2}^{\cdot}(t) u_{3}(t)\right] \\
& \quad-\frac{k_{\mathrm{ri}}}{2}\left[2 R I_{1}^{\cdot}(t) u_{0}(t)+2 R I_{0}(t) u_{1}(t)+R I_{2}(t) u_{1}(t)\right. \\
& \left.\quad+R I_{1}(t) u_{2}(t)+R I_{3}^{\cdot}(t) u_{2}(t)+R I_{2}(t) u_{3}(t)\right] \\
& \quad-K^{2}\left\{\left[D_{\mathrm{m}, 0}(t)-\frac{1}{2} D_{\mathrm{m}, 2}(t)\right] u_{1}(t)+\left[D_{\mathrm{m}, 1}(t)\right.\right. \\
& \left.\left.\quad-D_{\mathrm{m}, 3}(t)\right] u_{2}(t)-\frac{3}{2} D_{\mathrm{m}, 2}(t) u_{3}(t)\right\},
\end{aligned}
$$

where $S_{l}=\exp \left(-l^{2} K^{2} \sigma / 2\right)[6,21]$. For generality, in equation (28) we retain $D_{\mathrm{m}, 0}, D_{\mathrm{m}, 1}, D_{\mathrm{m}, 2}$ and $D_{\mathrm{m}, 3}$. However, in our simulations we assume $D_{\mathrm{m}}, j>0=0[9,21]$.

These coupled equations must be solved for the initial concentration values given in section 5 , i.e., when $t=0$ :

$$
\begin{gathered}
{\left[\mathrm{Dye}_{j \geqslant 0}^{*}\right]=\left[\begin{array}{ll}
H & D_{j \geqslant 0}^{\cdot}
\end{array}\right]=\left[R_{j \geqslant 0}^{\cdot}\right]=\left[M_{j \geqslant 0}^{\cdot}\right]} \\
=\left[R I_{j \geqslant 0}^{\cdot}\right]=\left[N_{j \geqslant 0}\right]=0, \quad \text { and }
\end{gathered}
$$

$\left[\right.$ Dye $\left._{j>0}\right]=\left[Z_{j>0}\right]=\left[u_{j>0}\right]=\left[E D_{j>0}\right]=\left[C T A_{j>0}\right]=0$.

\subsection{Refractive index modulation}

Kogelnik's two wave coupled wave theory [28] predicts that the diffraction efficiency of an unslanted volume transmission phase grating replayed on-Bragg is

$$
\eta=\sin ^{2}\left(\frac{\pi d n_{1}}{\lambda \cos \theta_{i n}}\right),
$$

where $\lambda$ is the replay wavelength, $\theta_{i n}$ is the on-Bragg angle inside the layer during the reconstruction step and $n_{1}$ is the amplitude of the first refractive index modulation harmonic.

In order to model the temporal evolution of the refractive index modulation, $n_{1}(t)$, which is determined by the behaviour of the photopolymer, it is necessary to know the refractive indices of the individual material components and their volume fractions. The temporal evolution of the average refractive index modulation in the layer can be determined using the Lorentz-Lorenz relation [21, 23, 26, 35, 36]

$$
\begin{gathered}
\frac{n^{2}-1}{n^{2}+2}=\phi^{(\mathrm{m})}(t) \frac{n_{\mathrm{m}}^{2}-1}{n_{\mathrm{m}}^{2}+2}+\phi^{(\mathrm{p})}(t) \frac{n_{\mathrm{p}}^{2}-1}{n_{\mathrm{p}}^{2}+2} \\
+\phi^{(\mathrm{b})}(t) \frac{n_{\mathrm{b}}^{2}-1}{n_{\mathrm{b}}^{2}+2}+\phi^{(\mathrm{H})}(t) \frac{n_{\mathrm{H}}^{2}-1}{n_{\mathrm{H}}^{2}+2},
\end{gathered}
$$

where $n_{\mathrm{m}}, n_{\mathrm{p}}, n_{\mathrm{b}}$ and $n_{\mathrm{H}}$ are the refractive indices of monomer, polymer, background material and holes, and $\phi^{(\mathrm{m})}$, $\phi^{(\mathrm{p})}, \phi^{(\mathrm{b})}$ and $\phi^{(\mathrm{H})}$ are the corresponding volume fractions of these species. During holographic grating formation, the volume fractions vary as a result of photochemical processes. During the polymerization of acrylamide, carbon double bonds are converted into carbon single bonds resulting in localized shrinkage [37] following the collapse of the holes generated [35]. Eventually, material mass transport by diffusion leads to swelling in the exposed areas [37]. However, in this paper we neglected all such shrinkage and 


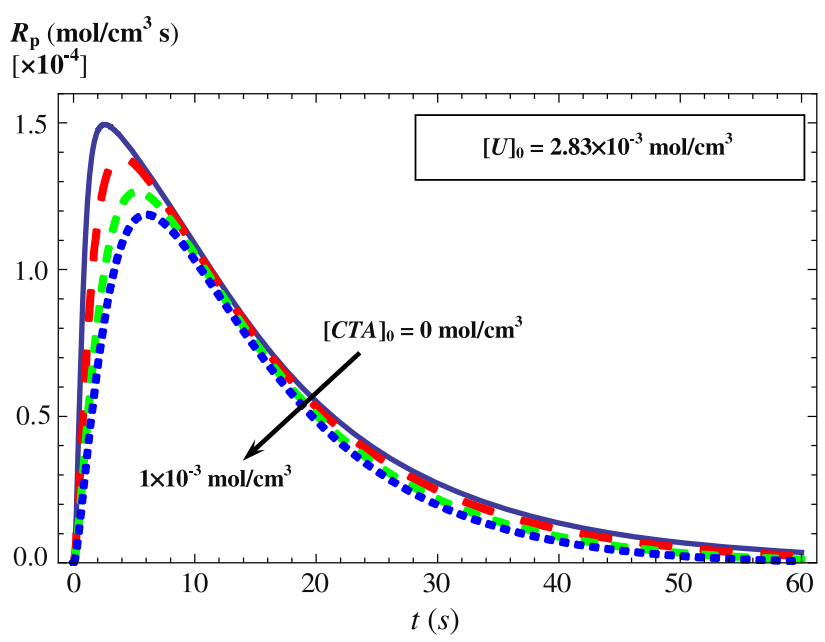

Figure 1. Simulation of the variation of polymerization rate, $R_{\mathrm{p}}$ ( $\mathrm{mol} \mathrm{cm} \mathrm{cm}^{-3} \mathrm{~s}^{-1}$ ), during exposure for four different initial concentrations of CTA, $[C T A]_{0}\left(\mathrm{~mol} \mathrm{~cm}^{-3}\right):\left\{0\right.$ (solid), $3 \times 10^{-4}$ (long dashed); $7 \times 10^{-4}$ (dashed) and $1 \times 10^{-3}$ (short dashed) $\}$.

swelling effects, and the hole concentration was assumed negligible $[21,35,38]$. In all the cases, the sum of the volume fractions of the individual components are assumed conserved:

$$
\phi^{(\mathrm{m})}(t)+\phi^{(\mathrm{p})}(t)+\phi^{(\mathrm{b})}(t)=1 .
$$

The temporal evolution of the refractive index modulation is expressed as [21, 23, 26]

$$
\begin{aligned}
n_{1}(t) & =\frac{\left(n_{\text {dark }}^{2}+2\right)^{2}}{6 n_{\text {dark }}}\left[\phi_{1}^{(\mathrm{m})}(t)\left(\frac{n_{\mathrm{m}}^{2}-1}{n_{\mathrm{m}}^{2}+2}-\frac{n_{\mathrm{b}}^{2}-1}{n_{\mathrm{b}}^{2}+2}\right)\right. \\
& \left.+\phi_{1}^{(\mathrm{p})}(t)\left(\frac{n_{\mathrm{p}}^{2}-1}{n_{\mathrm{p}}^{2}+2}-\frac{n_{\mathrm{b}}^{2}-1}{n_{\mathrm{b}}^{2}+2}\right)\right],
\end{aligned}
$$

where $n_{\text {dark }}$ is the refractive index of the photopolymer layer before exposure measured at the replay probe wavelength. $\phi_{1}^{(\mathrm{m})}(t)$ and $\phi_{1}^{(\mathrm{p})}(t)$ are the time varying first harmonic volume fraction components of monomer and polymer respectively [38]. Thus, $n_{1}(t)$ is predicted by combining equation (33) with the model described in section 3.

\section{Numerical results}

The predictions of the model presented in this paper are now discussed. All kinetic parameter values are assigned appropriate values, which are typical for the AA/PVA photopolymer material examined. The simulations are performed retaining four spatial concentration harmonics and therefore four coupled equations are solved for the initial conditions given in equation (29). In all cases, $[U]_{0}=2.83 \times$ $10^{-3} \mathrm{~mol} \mathrm{~cm}^{-3},[A]_{0}=1.22 \times 10^{-6} \mathrm{~mol} \mathrm{~cm}^{-3},[E D]_{0}=$ $3.18 \times 10^{-3} \mathrm{~mol} \mathrm{~cm}^{-3},[C T A]_{0}=1 \times 10^{-6} \mathrm{~mol} \mathrm{~cm}^{-3}$, and $[Z]_{0}=1 \times 10^{-8} \mathrm{~mol} \mathrm{~cm}^{-3}[8,22,23]$, where $[U]_{0}$, $[A]_{0},[E D]_{0},[C T A]_{0}$ and $[Z]_{0}$ represent the concentrations at $t=0$ of monomer, photosensitizer, electron donor, transfer agent and inhibitor, respectively. In this paper we assume that time varying viscosity effects are negligible, i.e., $D_{\mathrm{m}, j>0}=$ 0 , and that $D_{\mathrm{m} 0}=1.0 \times 10^{-10} \mathrm{~cm}^{2} \mathrm{~s}^{-1}[39,40]$. For an

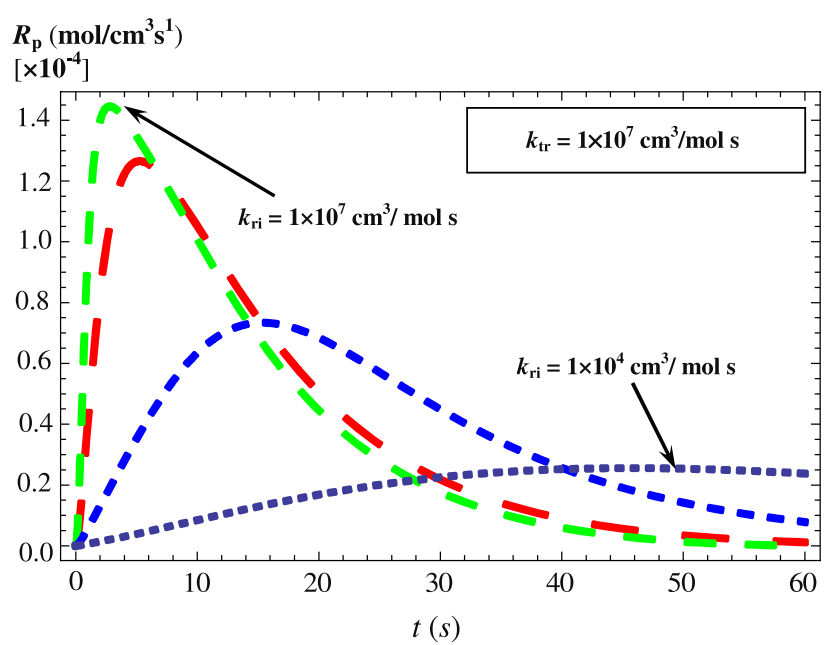

Figure 2. Simulation of the variation of polymerization rate, $R_{\mathrm{p}}$ $\left(\mathrm{s}^{-1}\right)$, during exposure for four different re-initiation rates, $k_{\mathrm{ri}}$ $\left(\mathrm{cm}^{3} \mathrm{~mol}^{-1} \mathrm{~s}^{-1}\right):\left\{1 \times 10^{4}\right.$ (very short dashed); $1 \times 10^{5}$ (short dashed); $1 \times 10^{6}$ (dashed) and $1 \times 10^{7}$ (long dashed) $\}$.

exposure intensity of $I_{0}=1 \mathrm{~mW} \mathrm{~cm}^{-2}, \lambda=532 \mathrm{~nm}$ and thickness $d=100 \mu \mathrm{m}$, the absorption parameters are, $\varepsilon=1.43 \times 10^{8} \mathrm{~cm}^{2} \mathrm{~mol}^{-1}, \phi=0.01 \mathrm{~mol} /$ Einstein and $T_{\mathrm{sf}}=0.76$. The oxygen diffusion coefficient is $D_{z}(x, t)=D_{z}=1.0 \times 10^{-8} \mathrm{~cm}^{2} \mathrm{~s}^{-1}$ and $\tau_{z}=0$, i.e., sealed layers are used [23]. The typical rate constants used were $k_{\mathrm{p}}=k_{\mathrm{i}}=1 \times 10^{7} \mathrm{~cm}^{3} \mathrm{~mol}^{-1} \mathrm{~s}^{-1}, k_{\mathrm{t}}=3 \times$ $10^{8} \mathrm{~cm}^{3} \mathrm{~mol}^{-1} \mathrm{~s}^{-1}, k_{\mathrm{tp}}=k t \times 10, k_{\mathrm{d}}=k_{\mathrm{b}}=1.6 \times$ $10^{3} \mathrm{~cm}^{3} \mathrm{~mol}^{-1} \mathrm{~s}^{-1}, k_{\mathrm{tr}}=1 \times 10^{7} \mathrm{~cm}^{3} \mathrm{~mol}^{-1} \mathrm{~s}^{-1}, k_{\mathrm{ri}}=$ $1 \times 10^{6} \mathrm{~cm}^{3} \mathrm{~mol}^{-1} \mathrm{~s}^{-1}, k_{z}=3 \times 10^{12} \mathrm{~cm}^{3} \mathrm{~mol}^{-1} \mathrm{~s}^{-1}$, and $k_{\mathrm{r}}=1.2 \times 10^{-3} \mathrm{~s}^{-1}[20,23,26,41]$. Assuming typical recording conditions for an unslanted transmission type volume holographic grating, i.e., period $\Lambda=400 \mathrm{~nm}$ and fringe visibility $V=1$, the resulting predictions of the temporal and spatial behaviour of the photochemical processes are now examined.

Figure 1 shows the polymerization rate, $R_{\mathrm{p}}$, which appears in equation (17), simulated for four different initial concentrations of CTA, $[C T A]_{0}=\left\{0,3 \times 10^{-4}, 7 \times 10^{-4}\right.$, and $\left.1 \times 10^{-3}\right\} \mathrm{mol} \mathrm{cm}^{-3}$. As the initial concentration of CTA is reduced to zero, it can be seen that the polymerization rate increases more rapidly towards a higher maximum value and then decreases at a faster rate. From figure 1, it should be noted that, for $[C T A]_{0}<[u]_{0}$, the effects of CTA on the polymerization rate are not very large, i.e., a similar number of monomer molecules will be consumed per unit time during grating formation.

The effects of various CTA re-initiation rates, $k_{\mathrm{ri}}$, on the polymerization rate are shown in figure 2 , where the rate constant of chain transfer is $k_{\mathrm{tr}}=1 \times 10^{7} \mathrm{~cm}^{3} \mathrm{~mol}^{-1} \mathrm{~s}^{-1}$ and four different re-initiation rates, $k_{\mathrm{ri}} \sim\left\{1 \times 10^{4}, 1 \times 10^{5}\right.$, $1 \times 10^{6}$ and $\left.1 \times 10^{7}\right\} \mathrm{cm}^{3} \mathrm{~mol}^{-1} \mathrm{~s}^{-1}$, are examined. Figure 2 clearly shows that the effect of a CTA on the polymerization rate is highly dependent on whether the re-initiation rate is comparable to the propagation rate, $k_{\mathrm{p}}=1 \times 10^{7}$ [20]. It can be seen that there is a large decrease in the polymerization rate, $R_{\mathrm{p}}$, as the re-initiation rate decreases. When the re-initiation 


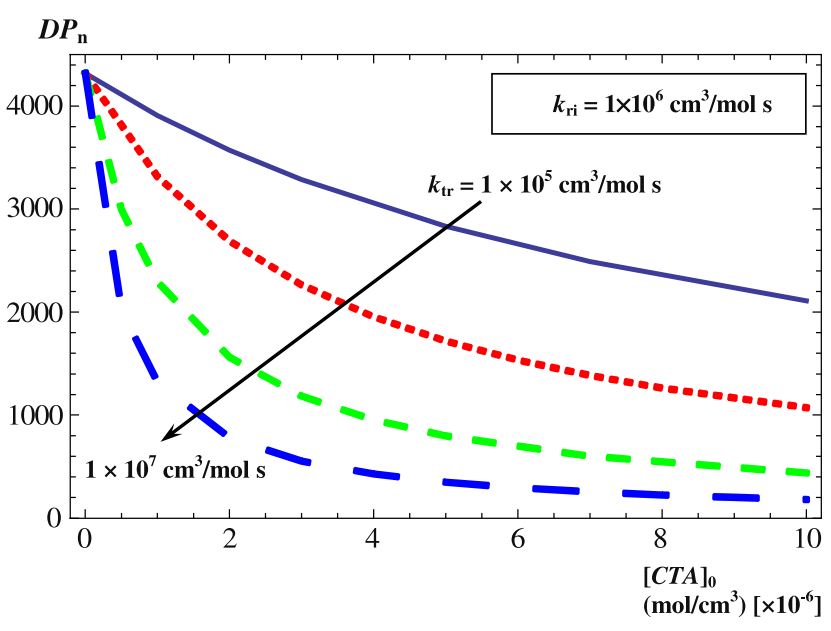

Figure 3. Effects of initial CTA concentration on the number-average degree of polymerization, $D P_{n}$, and contributions of various rate constants of chain transfer, $k_{\mathrm{tr}}\left(\mathrm{cm}^{3} \mathrm{~mol}^{-1} \mathrm{~s}^{-1}\right):\left\{1 \times 10^{5}\right.$ (solid); $3 \times 10^{5}$ (short dashed); $1 \times 10^{6}$ (dashed) and $1 \times 10^{7}$ (long dashed) $\}$.

rate, $k_{\mathrm{ri}}$, is comparable to the propagation rate, $k_{\mathrm{p}}$, one observes very little change in the polymerization rate. When the reinitiation rate, $k_{\mathrm{ri}}$, is slow compared to the propagation rate, i.e., when $k_{\mathrm{ri}} \ll k_{\mathrm{p}}$, one observes a large decrease in the polymerization rate, $R_{\mathrm{p}}$.

Different types of transfer agents will exhibit different kinetic behaviours, which result in variations in the polymerization rate and therefore changes to the numberaverage degree of polymerization, $D P_{n}$ [20]. The results in figure 3 demonstrate the effects of varying the initial CTA concentration on the $D P_{n}$ when the re-initiation rate $k_{\mathrm{r} i}=$ $1 \times 10^{6} \mathrm{~cm}^{3} \mathrm{~mol}^{-1} \mathrm{~s}^{-1}$. For a particular type of CTA, i.e., when $k_{\mathrm{tr}}=1 \times 10^{7} \mathrm{~cm}^{3} \mathrm{~mol}^{-1} \mathrm{~s}^{-1}$ (long dashed curve in figure 3), it can be seen that increasing the initial CTA concentration leads to a rapid decrease in $D P_{n}$ and that the $D P_{n}$ value decreases more slowly to the lowest value shown. This result indicates that, for an appropriate concentration of CTA, i.e., $1 \times 10^{-6}<[C T A]_{0}<4 \times 10^{-6} \mathrm{~mol} \mathrm{~cm}^{-3}, D P_{n}$ is always reduced with the inclusion of CTA and that the reduction is larger for higher CTA concentrations. Furthermore, when $k_{\text {tr }} \geqslant 1 \times 10^{7} \mathrm{~cm}^{3} \mathrm{~mol}^{-1} \mathrm{~s}^{-1}$, the model predicts that above some specific CTA concentration a threshold exists and further increases do not result in any further significant reduction in $D P_{n}$, i.e., when $[C T A]_{0}>4 \times 10^{-6} \mathrm{~mol} \mathrm{~cm}^{-3}$. Ideally, one wishes to identify the least amount of CTA required in order to achieve the largest reduction in the number-average of polymerization, $D P_{n}$. We also note that, for the same initial CTA concentration, a reduction in $D P_{n}$ also takes place for an increase in chain transfer kinetic value, $k_{\mathrm{tr}}$. Thus the addition of different types and concentrations of chain transfer agent is predicted to have different effects on the value of $D P_{n}$ and therefore on the average polymer chain length in the otherwise identical photopolymer system. As discussed in section 3, the non-local response length, $\sqrt{\sigma}$, and the number-average degree of polymerization, $D P_{n}$, are both related to the average polymer chain length. We would expect that any significant reduction in the number-average degree of polymerization,

$$
n_{1}\left[\times 10^{-3}\right]
$$

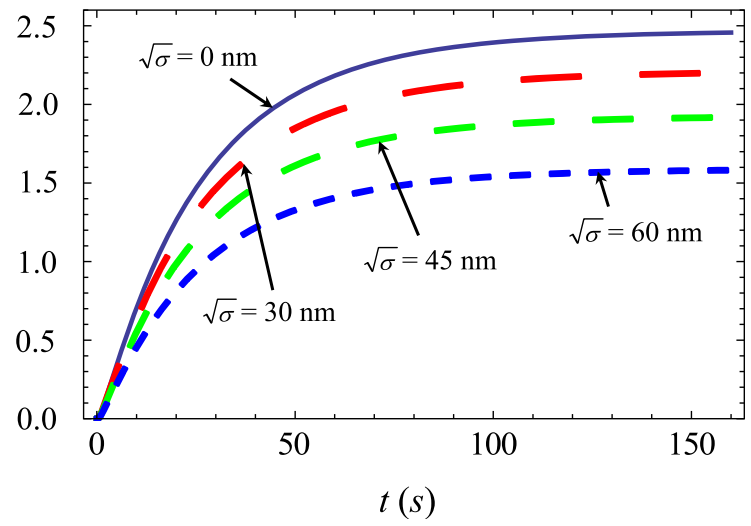

Figure 4. Simulations of the growth curves of refractive index modulation, $n_{1}$, at $\Lambda=400 \mathrm{~nm}$ for various values of the non-local response length, $\sqrt{\sigma}(\mathrm{nm})$ : $\{0$ (solid); 30 (long dashed); 45 (dashed) and 60 (short dashed) $\}$.

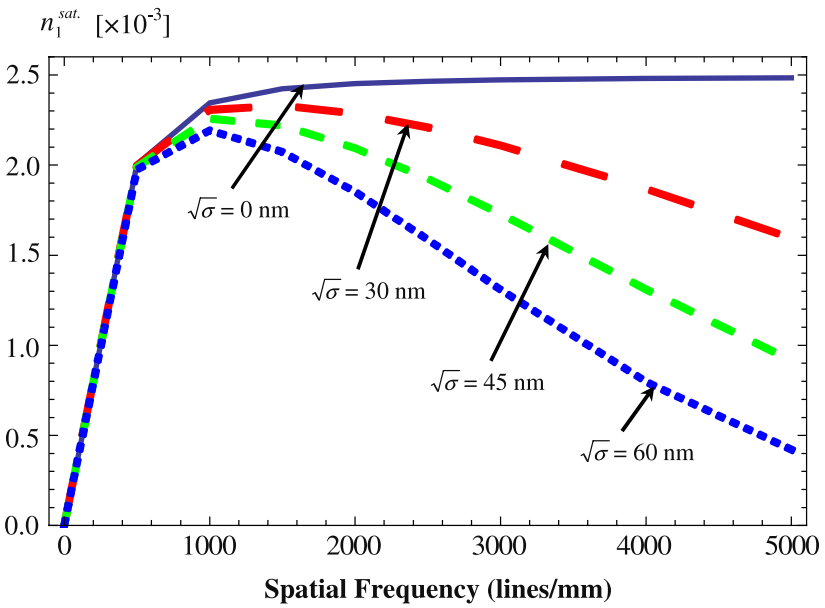

Figure 5. Simulations of the spatial frequency response of saturation refractive index modulation, $n_{1}$, for various values of the non-local response length, $\sqrt{\sigma}(\mathrm{nm}):\{0$ (solid); 30 (long dashed); 45 (dashed) and 60 (short dashed) .

$D P_{n}$, should be accompanied by a reduction in the non-local response length, $\sqrt{\sigma}$, and therefore by an improvement in the refractive index modulation, $n_{1}$, which can be recorded at high spatial frequencies in the material.

In order to demonstrate the relationship between $\sqrt{\sigma}$ and $n_{1}$, figure 4 shows four simulated growth curves of refractive index modulation, $n_{1}$, for four different values of $\sqrt{\sigma}$. In all cases the same typical rate constant values employed earlier in this section are used. We see that larger values of $\sqrt{\sigma}$ lead to lower saturation (maximum) values of $n_{1}$ for the same grating period. In other words, a lower $\sqrt{\sigma}$ value indicates that more localized polymerization, involving the generation of shorter polymer chains, takes place during holographic grating formation.

Figure 5 shows the saturation refractive index modulation, $n_{1}^{\text {sat }}$, plotted as a function of the grating spatial frequency, for the same grating parameter values as used in figure 4 . 
These results predict that lower $\sqrt{\sigma}$ values lead to a significant improvement in the high spatial frequency response of the material and therefore a reduction in the high spatial frequency roll-off observed experimentally. This is an important prediction of the NPDD model and motivates the study of the feasibility of applying chain transfer agents in free radical based photopolymer materials.

\section{Conclusion}

A detailed discussion of a kinetic model of free radical photopolymerization has been presented, which includes the major photochemical reactions and mass transport effects known to occur during the holographic grating formation process. This extended NPDD model, which includes the effects of chain transfer and re-initiation kinetics, has been applied to examine (i) the general effect of chain transfer agents on the photopolymer kinetic performance, (ii) the specific effects of chain transfer agent on the average polymer chain length formed, and (iii) the effects of the non-local spatial frequency response parameter on the photopolymer material.

In this paper, for the first time, an extended NPDD model is presented, which can be used to study the effects of CTAs. It has been shown that the model predicts that the average chain length formed can be controlled chemically in this way, and that optimum types and concentrations of CTAs can be identified. The extended NPDD model has also been shown to predict that as the non-local parameter decreases in size the high spatial frequency response of these material systems improves. For the free radical photopolymer system discussed, the non-local parameter has been associated with polymerization taking place due to polymer chain growth at points away from the point of initiation. Therefore, this is assumed to be an intimate link between the average polymer chain length, the polymer radius of gyration and the smearing effect during formation [31], which is quantified in the NPDD model by the non-local parameter, $\sigma$. Based on our results, it is reasonable to suggest that a detailed quantitative experimental study of the use of CTAs to control the spatial frequency response should be carried out. An experimental examination of a number of chain transfer agents is presented in part II of this series of papers. This experimental study involves various concentrations of a number of CTAs being added to the standard AA/PVA material and followed by the extraction of key kinetic parameter values by fitting the extended NPDD model presented in this paper, part I, to the resulting experimental data. The results support the use of CTAs to improve spatial frequency response in free radical photopolymerization systems.

\section{Acknowledgments}

One of the authors (JG) currently holds an IRCSET Government of Ireland Postgraduate Scholarship in Science, Engineering and Technology, while another author (MRG) currently holds a Government of Ireland Post-doctoral Scholarship in Science, Engineering and Technology, both granted by the Irish Research Council for Science, Engineering and Technology. We would also like to acknowledge the support of Enterprise Ireland and Science Foundation Ireland under the National Development Fund.

\section{References}

[1] Curtis K, Dhar L, Murphy L and Hill A 2010 Future Developments, in Holographic Data Storage: From Theory to Practical Systems (New York: Wiley)

[2] Ye C and McLeod R R 2008 GRIN lens and lens array fabrication with diffusion-driven photopolymer Opt. Lett. $332575-7$

[3] Sato A, Scepanovic M and Kostuk R 2003 Holographic edge-illuminated polymer Bragg gratings for dense wavelength division optical filters at $1550 \mathrm{~nm} \mathrm{Appl}$. Opt. 42 778-84

[4] Kashin O, Tolstik E, Matusevich V and Kowarschik R 2009 Numerical investigation of the $(1+1)$ D self-trapping of laser beams in polymeric films based on polymethylmethacrylate and phenanthrenequinone J. Opt. Soc. Am. B $262152-6$

[5] Bruder F and Facke T 2010 Materials in optical data storage J. Mater. Res. 101 199-215

[6] Sheridan J T and Lawrence J R 2000 Nonlocal response diffusion model of holographic recording in photopolymer J. Opt. Soc. Am. A 17 1108-14

[7] Lawrence J R, O’Neill F T and Sheridan J T 2002 Adjusted intensity nonlocal diffusion model of photopolymer grating formation J. Opt. Soc. Am. B 19 621-9

[8] Gleeson M R, Sabol D, Liu S, Close C E, Kelly J V and Sheridan J T 2008 Improvement of the spatial frequency response of photopolymer materials by modifying polymer chain length J. Opt. Soc. Am. B 25 396-406

[9] Zhao G H and Mouroulis P 1994 Diffusion-model of hologram formation in dry photopolymer materials J. Mod. Opt. 41 1929-39

[10] Kwon J H, Hwang H C and Woo K C 1999 Analysis of temporal behaviour of beams diffracted by volume gratings formed in photopolymers J. Opt. Soc. Am. B 16 1651-7

[11] Blaya S, Carretero L, Madrigal R F and Fimia A 2000 Theoretical model of holographic grating formation in photopolymerizable dry films in slanted geometry $O p t$. Commun. 173 423-33

[12] Kelly J V, O'Neill F T and Sheridan J T 2005 Cristian Neipp, Sergi Gallego, and Manuel Ortuno, 'holographic photopolymer materials: nonlocal polymerization-driven diffusion under nonideal kinetic conditions J. Opt. Soc. Am. B 22 407-16

[13] Gleeson M R, Kelly J V, Close C E, O'Neill F T and Sheridan J T 2006 Effects of absorption and inhibition during grating formation in photopolymer materials $\mathrm{J}$. Opt. Soc. Am. B 23 2079-88

[14] Mayo F R 1943 Chain transfer in the polymerization of styrene: the reaction of solvents with free radicals J. Am. Chem. Soc. 65 2324-9

[15] Goretta L A and Otremba R R 1981 US Patent Specification $4,307,215$

[16] Gartner H A 1992 US Patent Specification 5,171,783

[17] Fevola M, Hester R and McCormack C 2003 Molecular weight control of polyacrylamide with sodium formate as a chain-transfer agent: characterization via size exclusion chromatography/multi-angle laser light ccattering and determination of chain-transfer constant J. Polym. Sci. A $41560-8$

[18] Guo J, Gleeson M R, Liu S and Sheridan J T 2011 J. Opt. 13095602

[19] Lawrence J R, O'Neill F T and Sheridan J T 2001 Photopolymer holographic recording material Optik 112 449-63 
[20] Odian G 1991 Principles of Polymerization 4th edn (New York: Wiley)

[21] Gleeson M R and Sheridan J T 2009 Non-local photo-polymerization kinetics including multiple termination mechanisms and dark reactions: Part I. Modelling J. Opt. Soc. Am. B 26 1736-45

[22] Gleeson M R, Liu S, McLeod R R and Sheridan J T 2009 Non-local photo-polymerization kinetics including multiple termination mechanisms and dark reactions: Part II. Experimental validation J. Opt. Soc. Am. B 26 1746-54

[23] Gleeson M R, Liu S, Guo J and Sheridan J T 2010 Non-local photo-polymerization kinetics including multiple termination mechanisms and dark reactions: Part III. Primary radical generation and inhibition J. Opt. Soc. Am. B 27 1804-12

[24] Liu S, Gleeson M R, Guo J and Sheridan J T 2010 Optical characterization of photopolymers materials: theoretical and experimental examination of primary radical generation Appl. Phys. B 100 559-69

[25] Fimia A, Lopez N, Mateos F, Sastre R, Pineda J and Amat-Guerri F 1993 Elimination of oxygen inhibition in photopolymer systems used as holographic recording materials J. Mod. Opt. 40 699-706

[26] Liu S, Gleeson M R, Guo J and Sheridan J T 2010 High intensity response of photopolymer materials for holographic grating formation Macromolecules 43 9462-72

[27] Karasu F, Arsu N and Yagci Y 2007 2-mercapto thioxanthone as a chain transfer agent in free-radical polymerization J. Appl. Polym. Sci. 103 3766-70

[28] Kogelnik H 1969 Coupled wave theory for thick holographic gratings Bell Syst. Tech. J 48 2909-47

[29] O'Brien A K and Bowman C N 2006 Modelling the effect of oxygen on photopolymerization kinetics Macromol. Theory Simul. 15 176-82

[30] Guo J, Liu S, Gleeson M R and Sheridan J T 2011 Study of photosensitizer diffusion in a photopolymer material for holographic applications J. Opt. Eng. 50015801
[31] Doi M 1997 Introduction to Polymer Physics (Clarendon: Oxford) pp 1-8

[32] O'Neill F T, Lawrence J R and Sheridan J T 2002 Comparison of holographic photopolymer materials by use of analytic nonlocal diffusion models Appl. Opt. 41 845-52

[33] Valdebenito A and Encinas M V 2005 Thiophenols as chain transfer agents in the polymerization of vinyl monomers Polymer 46 10658-62

[34] Okaya T, Kikuchi K and Morii Y 1997 Polymerization of acrylamide in aqueous medium initiated with a redox system composed of cysteine and potassium bromate Macromol. Chem. Phys. 198 2027-34

[35] Aubrecht I, Miler M and Koudela I 1998 Recording of holographic diffraction gratings in photopolymers: theoretical modelling and real-time monitoring of grating growth J. Mod. Opt. 45 1465-77

[36] Pu H, Yin D, Gao B, Gao H, Dai H and Liu J 2009 Dynamic characterizations of high diffraction efficiency in volume Bragg grating formed by holographic photopolymerization J. Appl. Phys. 106083111

[37] O’Neill F T, Carr A J, Daniels S M, Gleeson M R, Kelly J V, Lawrence J R and Sheridan J T 2005 Refractive elements produced in photopolymer layers J. Mater. Sci. 40 4129-32

[38] Kelly J V, Gleeson M R, Close C E, O’Neill F T, Sheridan J T, Gallego S and Neipp C 2005 Temporal analysis of grating formation in photopolymer using the nonlocal polymerization-driven diffusion model Opt. Express 13 6990-7004

[39] Neipp C, Gallego S, Ortuno M, Marquez A, Alvarez M L, Belendez A and Pascual I 2003 First-harmonic diffusion-based model applied to a polyvinyl-alcohol-acrylamide-based photopolymer J. Opt. Soc. Am. B 20 2052-60

[40] Lougnot J, Jost P and Lavielle L 1997 Polymers for holographic recording: VI. Some basic ideas for modelling the kinetics of the recording process Pure Appl. Opt. 6 225-45

[41] Brandrup J, Immergut E H and Grulke E A 1999 Polymer Handbook 4th edn (New York: Wiley) 